CERN-TH.7539/94

hep-th/9412198

\title{
Topological Strings from WZW Models
}

\author{
K. Landsteiner, W. Lerche and A. Sevrin' \\ CERN, Geneva, Switzerland
}

\begin{abstract}
We show that the BRST structure of the topological string is encoded in the "small" $N=4$ superconformal algebra, enabling us to obtain, in a non-trivial way, the string theory from hamiltonian reduction of $A(1 \mid 1)$. This leads to the important conclusion that not only ordinary string theories, but topological strings as well, can be obtained, or even defined, by hamiltonian reduction from WZW models. Using two different gradations, we find either the standard $N=2$ minimal models coupled to topological gravity, or an embedding of the bosonic string into the topological string. We also comment briefly on the generalization to super Lie algebras $A(n \mid n)$.
\end{abstract}

CERN-TH.7539/94

December 1994

\footnotetext{
${ }^{1}$ Permanent address: Theoretische Natuurkunde, Vrije Universiteit Brussel, B-1050 Brussels, Belgium.
} 
It seems that the BRST structure of any string theory is encoded in a (twisted) $N=2$ supersymmetric extension of its gauge algebra. More precisely, the BRST structure of the bosonic string is characterized by a twisted $N=2$ superconformal algebra [1], that of the superstring by a twisted $N=3$ superconformal algebra [2, 3], that of $W_{n}$ strings by a twisted $N=2 W_{n}$ algebra [2], 4], etc. The idea is that one can add the BRST current and the anti-ghost to the gauge algebra, which then becomes superconformally enlarged. The BRST-charge itself is then one of the supercharges,

$$
\mathcal{Q}_{B R S T} \equiv G_{0}^{+}=\frac{1}{2 \pi i} \oint d z(c T+\ldots)
$$

while the anti-ghost $b(z)$ is the conjugate supercurrent, $G^{-}(z)$. This automatically ensures that $T(z)=\left\{\mathcal{Q}_{B R S T}, b(z)\right\}$.

Though this structure is quite general, it becomes especially important for non-critical strings. Here one can define the string theory in an almost completely algebraic way through quantum hamiltonian reduction [5] of an appropriate super-WZW model. In order to fully characterize the reduction, one has to specify a super-algebra and an embedding of $s l(2 \mid 1)$ into it. This already uniquely determines the specific extended superconformal algebra. Furthermore, one has to choose a particular gradation. This determines the particular free-field realization, which must be such that it allows for an interpretation in terms of string theory, ie., it must be of the form (11).

This approach of constructing string theories has the great advantage that the calculations are of algorithmic nature, enabling one to obtain the explicit form of the BRST operator in a relatively straightforward manner (straightforward at least compared to the usual trial and error method). Another advantage is that it also systematically produces a consistent set of screening operators, which are needed to properly define the free-field Hilbert space.

This program has been explicitly carried out for the non-critical $W_{n}$ strings, based on a reduction of $\operatorname{sl}(n \mid n-1)$ [2] and strings with $N$ supersymmetries, based on a reduction of $\operatorname{osp}(N+2 \mid 2)$ [3]. The generalization to arbitrary embeddings of $s l(2 \mid 1)$ in Lie superalgebras remains to be done, but we expect it to yield a classification of at least a very large class of non-critical string theories, if not of all of them. It has also recently been shown that one can revert the reduction in that it is possible to reconstruct the underlying Lie super algebra in terms of the field content of a string theory [6].

Up to now, however, it was not clear how to extend this program to topological strings [8]. The matter sector of a topological string is made up by a particular realization of the twisted $N=2$ algebra with central charge $c_{m}$. This topological conformal field theory is coupled to topological gravity, which can most easily be represented by a supersymmetric ghost system consisting of diffeomorphism ghosts, $b(z), c(z)$, and their bosonic super- 
partners, $\beta(z), \gamma(z)[7]$. Out of these ghost systems, one can construct a twisted $N=2$ algebra with topological central charge equal to -9 . Since the "true" central charge of each building block vanishes identically and separately (as implied by the twisting), the BRST-operator automatically squares to zero independent from whether the topological central charges add up to zero or not. Thus, there is no need to include an extra Liouville sectorf. In that sense there is no "critical" central charge for topological strings. Nevertheless, it is well-known that topological strings with $c_{m}=9$ have special properties [9], and indeed, in our construction given further below, this "critical" case of topological strings will turn out to be distinguished.

In this letter we complete our program of obtaining all known string theories from WZW models, by showing how topological strings can be constructed from a suitable reduction of $s l(2 \mid 2)$. The possibility of choosing different gradations will enable us to find two different free-field realizations of the "small" $N=4$ algebra. Both have interesting physical interpretations.

The first gradation results in $N=2$ minimal models coupled to topological gravity, represented by a fermionic and a bosonic ghost-system, each with spins $(2,-1)$.

The second gradation results in an embedding of the non-critical bosonic string into the topological string, in the sense that the matter system is represented by the bosonic string. That is, we will not only go one step further in the program initiated in [3] of classifying string theories by purely Lie-algebraic methods, but we also obtain a new mechanism of a string embedding that is defined intrinsically in terms of hamiltonian reduction. This embedding looks different from the kind of string embeddings developed in [10, 11, where one embeds string theories with $N$ supersymmetries into strings with $N+1$ supersymmetries; no obvious connection to hamiltonian reduction could be made so far for these embeddings.

Let us now describe the BRST-operator structure of topological strings is some more detail. Neglecting the currents from the Liouville sector, the total BRST charge, $Q_{B R S T}=$ $Q_{s}+Q_{v}$, consists of the following two contributions [8]:

$$
\begin{aligned}
Q_{s} & =\oint\left(G_{+m}+G_{+g h}\right) \\
Q_{v} & =\oint\left(c\left(T_{m}+\frac{1}{2} T_{g h}\right)+\gamma\left(G_{-m}+\frac{1}{2} G_{-g h}\right)\right) .
\end{aligned}
$$

This implies that $T(z)$ is conjugate to $b(z)$ with respect to $Q_{v}: T(z) \propto\left\{Q_{v}, b(z)\right\}$, and to $G_{-}(z)$ with respect to $Q_{s}: T(z) \propto\left\{Q_{s}, G_{-}(z)\right\}$, and suggests that the BRST structure of the topological string should be related to a doubly twisted, $N=4$ superconformal

\footnotetext{
${ }^{2}$ It has been shown [8], however, that the inclusion of a Liouville sector makes the computation of physical observables more feasible
} 
algebra 12, 3].

There are essentially two $N=4$ algebras, a "large" one [13], which is obtained from the hamiltonian reduction of $D(2,1, \alpha)$, and a "small" one [14], obtained from the reduction of $s l(2 \mid 2)$ (or $A(1 \mid 1)$ in a different notation) [15]. In [3], topological strings were related to a subalgebra of the large $N=4$ algebra. However, no obvious connection with the reduction of $D(2,1, \alpha)$ could be found. As we will now argue, the more appropriate structure to look at is the small $N=4$ algebra, which is generated by the energy-momentum tensor, four supercurrents and an $s u(2)$ affine Lie algebra.

Therefore our starting point is the Lie super algebra $\operatorname{sl}(2 \mid 2)$. It consists of two $\operatorname{sl}(2)$ affine algebras whose currents will be denoted by $E^{i}(z)$ and $J^{i}(z)$, with $i \in\{+, 0,-\}$. The fermionic currents transform according to the $(2 \mid \overline{2}) \oplus(\overline{2} \mid 2)$ representation of the $\operatorname{sl}(2) \oplus \operatorname{sl}(2)$ subalgebra. We thus denote the fermionic currents by $j_{a}^{i j}(z)$, with $a, i, j \in\{+,-\}$. The OPE's are explicitly given byf

$$
\begin{array}{cc}
E^{0}\left(z_{1}\right) E^{0}\left(z_{2}\right)=\frac{\kappa}{8} z_{12}^{-2}, & J^{0}\left(z_{1}\right) J^{0}\left(z_{2}\right)=-\frac{\kappa}{8} z_{12}^{-2}, \\
E^{0}\left(z_{1}\right) E^{ \pm}\left(z_{2}\right)= \pm z_{12}^{-1} \frac{1}{2} E^{ \pm}\left(z_{2}\right), & J^{0}\left(z_{1}\right) J^{ \pm}\left(z_{2}\right)= \pm z_{12}^{-1} \frac{1}{2} J^{ \pm}\left(z_{2}\right), \\
E^{+}\left(z_{1}\right) E^{-}\left(z_{2}\right)=\frac{\kappa}{4} z_{12}^{-2}+z_{12}^{-1} E^{0}\left(z_{2}\right), & J^{+}\left(z_{1}\right) J^{-}\left(z_{2}\right)=-\frac{\kappa}{4} z_{12}^{-2}+z_{12}^{-1} J^{0}\left(z_{2}\right), \\
E^{0}\left(z_{1}\right) j_{a}^{ \pm i}\left(z_{2}\right)= \pm \frac{1}{4} z_{12}^{-1} j_{a}^{ \pm i}\left(z_{2}\right), & E^{ \pm}\left(z_{1}\right) j_{a}^{\mp i}\left(z_{2}\right)=\frac{1}{2} z_{12}^{-1} j_{a}^{ \pm i}\left(z_{2}\right), \\
J^{0}\left(z_{1}\right) j_{a}^{i \pm}\left(z_{2}\right)= \pm \frac{1}{4} z_{12}^{-1} j_{a}^{i \pm}\left(z_{2}\right), & J^{ \pm}\left(z_{1}\right) j_{a}^{i \mp}\left(z_{2}\right)=\frac{1}{2} z_{12}^{-1} j_{a}^{i \pm}\left(z_{2}\right), \\
j_{+}^{ \pm \pm}\left(z_{1}\right) j_{-}^{\mp \mp}\left(z_{2}\right)=\frac{\kappa}{4} z_{12}^{-2} \pm \frac{1}{2} z_{12}^{-1}\left(E^{0}\left(z_{2}\right)-J^{0}\left(z_{2}\right)\right) \\
j_{+}^{ \pm \mp}\left(z_{1}\right) j_{-}^{\mp \pm}\left(z_{2}\right)=-\frac{\kappa}{4} z_{12}^{-2} \mp \frac{1}{2} z_{12}^{-1}\left(E^{0}\left(z_{2}\right)+J^{0}\left(z_{2}\right)\right) \\
j_{+}^{ \pm \pm}\left(z_{1}\right) j_{-}^{ \pm \mp}\left(z_{2}\right)=-\frac{1}{2} z_{12}^{-1} E^{ \pm}\left(z_{2}\right),
\end{array}
$$

The relevant embedding of $\operatorname{sl}(2 \mid 1)$ into $\operatorname{sl}(2 \mid 2)$ is given by choosing the bosonic generators of $s l(2 \mid 1)$ to be $E^{i}$ and $J^{0}$. Note that we still have two possibilities for choosing the fermionic part of the embedding. This traces back to the curious property of $A(1 \mid 1)$ that the fermionic roots are simultaneously both, positive and negative. We will see in the following that this does not really constitute an ambiguity.

\footnotetext{
${ }^{3}$ We used the metric conventions $g_{00}^{E}=-g_{00}^{J}=-4, g_{+-}^{E}=-g_{+-}^{J}=-2$ and $g_{++--}^{+-}=-2, g_{+--+}^{-+}=$ $-2, g_{++--}^{-+}=2, g_{+--+}^{+-}=2$.
} 
As was emphasized in [3], the embedding just determines the kind of superconformal algebra. However, in order to obtain a specific free-field realization of this algebra that allows for a string interpretation, one has to choose the gradation appropriately. The gradation will determine a consistent set of first class constraints on the negatively graded part, and these constraints imply the presence of a gauge symmetry. The gauge fixing and the construction of the generators of the conformal algebra proceed then in a way quite similar to what was developed in [16]. That is, although the algebra is independent of the gradation, its realization, and in particular the Miura transformation, depends on it.

We now apply this to $s l(2 \mid 2)$. The standard gradation, i.e. the one used in [16], is given by the eigenvalues of $\operatorname{ad}_{2 E^{0}}$. However, this gives rise to a "symmetric" free-field realization that has no interpretation as a string BRST algebra. In order to find appropriate gradations, we note that in the classification [17] of Lie super-algebras one distinguishes between algebras of type 2, whose fermionic part carries an irreducible representation of the bosonic subalgebra, and algebras of type 1 , where this does not hold. Type 1 super Lie-algebras admit a canonical gradation of the form $g=g_{-1 / 2} \oplus g_{0} \oplus g_{1 / 2}$, where both $g_{1 / 2}$ and $g_{-1 / 2}$ are irreducible representations of $g_{0}$. In our case we have $j_{+}^{i j} \in g_{+1 / 2}$ and $j_{-}^{i j} \in g_{-1 / 2}$, while the remainder of the generators belong to $g_{0}$.

We find that we can use two different gradations, denoted by $I$ and $I I$, to describe sensible, string-type free-fields realizations. Gradation $I$ is given by the sum of the canonical gradation with the gradation defined by the action of $\operatorname{ad}_{2\left(E^{0}+J^{0}\right)}$, whereas gradation $I I$ is defined by the eigenvalues of $\operatorname{ad}_{\left(2 E^{0}+4 J^{0}\right)}$ ignoring the canonical one. In the following table, we explicitly give the corresponding grades for the various elements of $s l(2 \mid 2)$ :

\begin{tabular}{|c||c|c|c||c|c|c||c|c|c|c|c|c|c|c|}
\hline & $E^{+}$ & $E^{0}$ & $E^{-}$ & $J^{+}$ & $J^{0}$ & $J^{-}$ & $j_{+}^{++}$ & $j_{+}^{+-}$ & $j_{+}^{-+}$ & $j_{+}^{--}$ & $j_{-}^{++}$ & $j_{-}^{+-}$ & $j_{-}^{-+}$ & $j_{-}^{--}$ \\
\hline$I$ & 1 & 0 & -1 & 1 & 0 & -1 & $\frac{3}{2}$ & $\frac{1}{2}$ & $\frac{1}{2}$ & $-\frac{1}{2}$ & $\frac{1}{2}$ & $-\frac{1}{2}$ & $-\frac{1}{2}$ & $-\frac{3}{2}$ \\
\hline$I I$ & 1 & 0 & -1 & 2 & 0 & -2 & $\frac{3}{2}$ & $-\frac{1}{2}$ & $\frac{1}{2}$ & $-\frac{3}{2}$ & $\frac{3}{2}$ & $-\frac{1}{2}$ & $\frac{1}{2}$ & $-\frac{3}{2}$ \\
\hline
\end{tabular}

Let us now first give a detailed discussion of the hamiltonian reduction of $s l(2 \mid 2)$ using gradation $I$. The constraints we impose on the negatively graded currents are of the form $\Phi^{\alpha}=0$ with

$$
\begin{array}{rlrl}
\Phi_{E}^{-} & =E^{-}-\frac{\kappa}{2}, & & \Phi_{J}^{-}=J^{-}-\mu, \\
\Phi_{-}^{+-}=j_{-}^{+-}-\psi, & \Phi_{+}^{--}=j_{+}^{--}-\tau+\frac{1}{2} \bar{\psi} \mu, \\
\Phi_{-}^{-+}=j_{-}^{-+}-\bar{\tau}, & \Phi_{-}^{--}=j_{-}^{--} .
\end{array}
$$

The auxiliary fields, $(\mu, \psi)$, and their conjugates, $(\bar{\mu}, \bar{\psi})$, were introduced in order to avoid 
that currents which are in the kernel of $\operatorname{ad}_{E^{+}}$and would become the leading term of the conformal currents, are constrained to zero (see [3] for more details). The additional auxiliary fields $(\tau, \bar{\tau})$ are needed to ensure that all constraints are first class.

The constrained theory derives from the actionf

$$
S_{i n v}=\kappa S_{W Z W}[g]+\frac{1}{\pi} \int d^{2} z \operatorname{str}(\bar{A} \Phi)-\frac{1}{2 \pi} \int d^{2} z(\bar{\psi} \bar{\partial} \psi-\bar{\mu} \bar{\partial} \mu)-\frac{2}{\kappa \pi} \int d^{2} z \bar{\tau} \bar{\partial} \tau
$$

where $S_{W Z W}$ is the Wess-Zumino-Witten action on $S L(2 \mid 2)$ and the gauge fields $\bar{A}$ appear as Lagrange multipliers that impose the constraints. The gauge symmetry of the action is generated by the strictly positively graded subalgebra of $\operatorname{sl}(2 \mid 2)$ :

$$
\begin{array}{rlrl}
\delta \bar{A}=\bar{\partial} \eta+[\bar{A}, \eta], & & \delta J=\partial \eta+[J, \eta] \\
\delta \mu=0, & & \delta \bar{\mu}=2 \eta_{J}^{+}+\eta_{-}^{++} \bar{\psi} \\
\delta \psi=-\eta_{-}^{++} \mu & , & \delta \bar{\psi}=-2 \eta_{+}^{-+} \\
\delta \tau=\frac{\kappa}{2} \eta_{+}^{+-}, & \delta \bar{\tau}=\frac{\kappa}{2} \eta_{-}^{++} .
\end{array}
$$

The kinetic terms of the auxiliary fields follow from requiring gauge invariance and give rise to the following OPE's

$$
\psi\left(z_{1}\right) \cdot \bar{\psi}\left(z_{2}\right)=\mu\left(z_{1}\right) \cdot \bar{\mu}\left(z_{2}\right)=z_{12}^{-1} \quad \tau\left(z_{1}\right) \cdot \bar{\tau}\left(z_{2}\right)=\frac{\kappa}{4} z_{12}^{-1} .
$$

We now quantize this theory by first fixing the gauge symmetry by setting $\bar{A}=0$, and introducing the ghosts $C$ and anti-ghosts $B$ :

$$
\begin{aligned}
& C=c_{E}^{+} t_{+}^{E}+c_{J}^{+} t_{+}^{J}+\gamma_{+}^{-+} t_{-+}^{+}+\gamma_{-}^{++} t_{++}^{-}+\gamma_{+}^{+-} t_{+-}^{+}+\gamma_{+}^{++} t_{++}^{+} \\
& B=b_{E}^{-} t_{-}^{J}+b_{J}^{-} t_{-}^{J}+\beta_{-}^{+-} t_{+-}^{-}+\beta_{+}^{--} t_{--}^{+}+\beta_{-}^{-+} t_{-+}^{-}+\beta_{-}^{--} t_{--}^{-} .
\end{aligned}
$$

The gauge fixing action reads

$$
S_{g . f .}=\frac{1}{2 \pi} \int d^{2} z(D \bar{A}+B \bar{\partial} C+\bar{A}\{B, C\}),
$$

where $D$ is a Langrange multiplier that imposes the gauge condition. From this we can read off the OPE's for the ghosts

$$
\begin{gathered}
b_{E}^{-}\left(z_{1}\right) \cdot c_{E}^{+}\left(z_{2}\right)=-b_{J}^{-}\left(z_{1}\right) \cdot c_{J}^{+}\left(z_{2}\right)=-\beta_{+}^{+-}\left(z_{1}\right) \cdot \gamma_{-}^{-+}\left(z_{2}\right)= \\
=-\beta_{+}^{--}\left(z_{1}\right) \cdot \gamma_{-}^{++}\left(z_{2}\right)=-\beta_{-}^{-+}\left(z_{1}\right) \cdot \gamma_{+}^{+-}\left(z_{2}\right)=\beta_{-}^{--}\left(z_{1}\right) \cdot \gamma_{+}^{++}\left(z_{2}\right)=z_{12}^{-1} .
\end{gathered}
$$

The BRST-charge then takes the standard form:

$$
\mathcal{Q}=\frac{1}{2 \pi i} \oint \operatorname{str}\left(C \Phi+\frac{1}{2} C J_{g h}\right),
$$

\footnotetext{
${ }^{4}$ The supertrace is defined by $\operatorname{str}(x y)=x^{a} y^{b} g_{a b}(-1)^{\operatorname{deg}(b)}$, where $\operatorname{deg}(b)=0$ or 1 , if it corresponds to a bosonic or fermionic generator.
} 
where the ghost currents, $J_{g h}=\frac{1}{2}\{B, C\}$, are given by:

$$
\begin{aligned}
E_{g h}^{+} & =-\frac{1}{2} \beta_{-}^{+-} \gamma_{+}^{++}, \quad E_{g h}^{-}=\frac{1}{2} \beta_{-}^{--} \gamma_{+}^{-+}, \\
E_{g h}^{0} & =-\frac{1}{2} b_{E}^{-} c_{E}^{+}+\frac{1}{4} \beta_{-}^{+-} \gamma_{+}^{-+}-\frac{1}{4} \beta_{+}^{--} \gamma_{-}^{++}-\frac{1}{4} \beta_{-}^{-+} \gamma_{+}^{+-}+\frac{1}{4} \beta_{-}^{--} \gamma_{+}^{++}, \\
J_{g h}^{+} & =-\frac{1}{2} \beta_{-}^{-+} \gamma_{+}^{++}, \quad J_{g h}^{-}=\frac{1}{2} \beta_{-}^{--} \gamma_{+}^{+-}, \\
J_{g h}^{0} & =\frac{1}{2} b_{J}^{-} c_{J}^{+}-\frac{1}{4} \beta_{-}^{+-} \gamma_{+}^{-+}-\frac{1}{4} \beta_{+}^{--} \gamma_{-}^{++}+\frac{1}{4} \beta_{-}^{-+} \gamma_{+}^{+-}+\frac{1}{4} \beta_{-}^{--} \gamma_{+}^{++}, \\
j_{+g h}^{+-} & =-\frac{1}{2} b_{J}^{-} \gamma_{+}^{++}+\frac{1}{2} \beta_{+}^{--} c_{E}^{+}, \quad j_{+g h}^{--}=\frac{1}{2} b_{E}^{-} \gamma_{+}^{+-}-\frac{1}{2} b_{J}^{-} \gamma_{+}^{-+}, \\
j_{+g h}^{-+} & =\frac{1}{2} b_{E}^{-} \gamma_{+}^{++}-\frac{1}{2} \beta_{+}^{--} c_{J}^{+}, \quad j_{-g h}^{++}=-\frac{1}{2} \beta_{-}^{+-} c_{J}^{+}+\frac{1}{2} \beta_{-}^{-+} c_{E}^{+}, \\
j_{-g h}^{+-} & =-\frac{1}{2} b_{J}^{-} \gamma_{-}^{++}+\frac{1}{2} \beta_{-}^{--} c_{E}^{+}, \quad j_{-g h}^{-+}=\frac{1}{2} b_{E}^{-} \gamma_{-}^{++}-\frac{1}{2} \beta_{-}^{--} c_{J}^{+} .
\end{aligned}
$$

We now proceed by closely following the methods of ref. [16]. Classically, the generators of the extended conformal algebra are given by the gauge invariant polynomials. At the quantum level this translates to the fact the generators of the conformal algebra are given by the generators of the cohomology of $\mathcal{Q}$ on the algebra $\mathcal{A}$, which consists of all normal ordered products of $\left\{\hat{J} \equiv J+J_{g h}, C, B, \tau, \bar{\tau}, \psi, \bar{\psi}, \mu, \bar{\mu}\right\}$ and their derivatives. The computation of the cohomology is not very hard due to the presence of a double gradation of the complex $\mathcal{A}$ :

$$
\mathcal{A}=\bigoplus_{\substack{m, n \in \frac{1}{2} \mathbf{Z} \\ m+n \in \mathbf{Z}}} \mathcal{A}_{(m, n)}
$$

The grade of the various fields is given by $(m, n)$, where $m$ is the grade previously given in the table and $m+n$ is the ghost number. The auxiliary fields have grade $(0,0)$. The BRST operator splits into three parts, each of which has a definite grade:

$$
\mathcal{Q}=\mathcal{Q}_{0}+\mathcal{Q}_{1}+\mathcal{Q}_{2}
$$

where

$$
\begin{aligned}
\mathcal{Q}_{0}= & \frac{1}{2 \pi i} \oint \frac{\kappa}{2} c_{E}^{+}-c_{J}^{+} \mu \\
\mathcal{Q}_{1}= & \frac{1}{2 \pi i} \oint \gamma_{+}^{-+} \psi+\gamma_{-}^{++}\left(\tau-\frac{1}{2} \bar{\psi} \mu\right)+\gamma_{+}^{+-} \tau, \\
\mathcal{Q}_{2}= & \frac{1}{2 \pi i} \oint-c_{E}^{+}\left(E^{-}+\frac{1}{2} E_{g h}^{-}\right)+c_{J}^{+}\left(J^{-}+\frac{1}{2} J_{g h}^{-}\right)-\gamma_{+}^{-+}\left(j_{-}^{+-}+\frac{1}{2} j_{-g h}^{+-}\right)- \\
& -\gamma_{-}^{++}\left(j_{+}^{--}+\frac{1}{2} j_{+g h}^{--}\right)-\gamma_{+}^{+-}\left(j_{-}^{-+}+\frac{1}{2} j_{-g h}^{-+}\right)+\gamma_{+}^{++} j_{-}^{--},
\end{aligned}
$$

which have grade $(1,0),(1 / 2,1 / 2)$ and $(0,1)$, respectively.

Using the fact that $B$ and $\hat{\Phi}$ (where $\hat{\Phi}$ denotes the substitution of $J$ by $\hat{J}$ in the constraints 
(4)) generate a sub-complex, one can argue along lines similar to those in [16 that the cohomology of $\mathcal{Q}$ on $\mathcal{A}$ is isomorphic to the one computed on a reduced complex $\widehat{\mathcal{A}}$. The reduced complex is generated by those fields of $\mathcal{A}$ which have grades $(m, n)$ with $m \geq 0$. We thus introduce a filtration $\widehat{\mathcal{A}}^{m}, m \in \frac{1}{2} \mathbf{Z}$ of $\widehat{\mathcal{A}}$ :

$$
\widehat{\mathcal{A}}^{m} \equiv \bigoplus_{k \in \frac{1}{2} \mathbf{Z} l \geq m} \bigoplus_{\mathcal{\mathcal { A }}_{(k, l)}}
$$

This leads to a spectral sequence $\left(E_{r}, d_{r}\right), r \geq 1$, converging to $H^{*}(\widehat{\mathcal{A}} ; \mathcal{Q})$, where we have $E_{r}=H^{*}\left(E_{r-1} ; d_{r-1}\right)$. The sequence collapses already after the second step: $E_{1}=$ $H^{*}\left(\widehat{\mathcal{A}}, \mathcal{Q}_{0}\right), E_{2}=E_{\infty}=H^{*}\left(E_{1}, \mathcal{Q}_{1}\right)$, where $E_{2} \simeq H^{*}(\widehat{\mathcal{A}} ; \mathcal{Q})$ is generated by $\left\{\hat{E}^{+}, \hat{J}^{+}, \hat{J}^{0}+\right.$ $\left.\frac{1}{2} \mu \bar{\mu}-\frac{1}{4} \bar{\psi} \psi-\frac{1}{\kappa} \tau \bar{\tau}, \mu, \hat{j}_{+}^{++}, \hat{j}_{-}^{++}, \hat{j}_{+}^{+-}+\frac{2}{\kappa} \hat{j}_{+}^{-+} \mu, \psi+\frac{2}{\kappa} \bar{\tau} \mu\right\}$. The explicit form of the generators now follows from a tic-tac-toe procedure and have the structure:

$$
\Lambda=\Lambda^{(m,-m)}+\Lambda^{\left(m-\frac{1}{2},-m+\frac{1}{2}\right)}+\ldots+\Lambda^{(0,0)}
$$

where the upper indices refer to the grades of the individual terms and the leading terms $\Lambda^{(m,-m)}$ are the generators of $E_{2}$ given above. Using exactly the same arguments as those in [16], one can show that $\Lambda \rightarrow \Lambda^{(0,0)}$ is an algebra isomorphism, which is nothing but the quantum Miura transformation. This provides us almost with the desired free-field realization. In order to obtain the correct realization, we need in addition to perform a similarity transformation (see also [3]) given by $\Lambda^{(0,0) \prime}=S \Lambda^{(0,0)} S^{-1}$, where $S=\exp \left[\frac{1}{\kappa \pi i} \oint d z \mu \bar{\psi} \bar{\tau}\right]$. Bosonizing the Cartan currents

$$
\hat{E}^{0}=\frac{i \sqrt{\kappa}}{4} \partial(\bar{\varphi}-\varphi), \quad \hat{J}^{0}=\frac{i \sqrt{\kappa}}{4} \partial(\bar{\varphi}+\varphi), \quad \varphi\left(z_{1}\right) \cdot \bar{\varphi}\left(z_{2}\right)=\ln \left(z_{12}\right),
$$

and rescaling the auxiliary fields, $\tau \rightarrow \frac{i \sqrt{\kappa}}{2} \tau, \quad \bar{\tau} \rightarrow \frac{-i \sqrt{\kappa}}{2} \bar{\tau}$, then finally yields the following currents of the BRST algebra:

$$
\begin{aligned}
T= & -\partial \varphi \partial \bar{\varphi}+\frac{i \sqrt{\kappa}}{2}\left(\partial^{2} \varphi+\partial^{2} \bar{\varphi}\right)-\frac{1}{2} \tau \partial \bar{\tau}+\frac{1}{2} \partial \tau \bar{\tau}+ \\
& +\mu \partial \bar{\mu}-\frac{3}{2} \psi \partial \bar{\psi}-\frac{1}{2} \partial \psi \bar{\psi} \\
G_{+}= & \partial \bar{\varphi} \bar{\tau}+\psi \bar{\mu}-i \sqrt{\kappa} \partial \bar{\tau} \\
G_{-}= & -\partial \varphi \tau+\bar{\psi} \partial \mu+2 \partial \bar{\psi} \mu+i \sqrt{\kappa} \partial \tau, \\
\hat{G}_{+}= & \bar{\psi}\left(-\partial \varphi \partial \bar{\varphi}-\tau \partial \bar{\tau}+i \sqrt{\kappa} \partial^{2} \bar{\varphi}+\mu \partial \bar{\mu}+\frac{1}{2} \partial \mu \bar{\mu}-\psi \partial \bar{\psi}\right)- \\
& -\bar{\mu}\left(-\partial \bar{\varphi} \tau+i \sqrt{\kappa} \partial \tau+\partial \bar{\psi} \mu+\frac{1}{2} \bar{\psi} \partial \mu\right)- \\
& -\partial(\bar{\psi}(i \sqrt{\kappa} \partial \bar{\varphi}-i \sqrt{\kappa} \partial \varphi-\tau \bar{\tau}+\mu \bar{\mu}))-(1+\kappa) \partial^{2} \bar{\psi} \\
\hat{G}_{-}= & \psi \\
K_{+}= & -\bar{\psi} \bar{\tau} \partial \bar{\varphi}+i \sqrt{\kappa} \bar{\psi} \partial \bar{\tau}+\bar{\mu} \psi \bar{\psi}-\bar{\mu}^{2} \mu+\bar{\mu} \tau \bar{\tau}-i \sqrt{\kappa} \bar{\mu} \partial \bar{\varphi}+
\end{aligned}
$$




$$
\begin{aligned}
& +i \sqrt{\kappa} \bar{\mu} \partial \varphi-(1+\kappa) \partial \bar{\mu} \\
K_{3}= & i \sqrt{\kappa}(\partial \bar{\varphi}-\partial \varphi)+2 \mu \bar{\mu}-\psi \bar{\psi}-\tau \bar{\tau} \\
K_{-}= & \mu .
\end{aligned}
$$

They are canonically normalized such that they generate the small $N=4$ algebra:

$$
\begin{aligned}
G_{+}\left(z_{1}\right) G_{-}\left(z_{2}\right) & =\hat{G}_{+}\left(z_{1}\right) \hat{G}_{-}\left(z_{2}\right)=\frac{c}{3} z_{12}^{-3}+z_{12}^{-2} K_{3}\left(z_{2}\right)+z_{12}^{-1}\left(T+\frac{1}{2} \partial K_{3}\right)\left(z_{2}\right) \\
G_{ \pm}\left(z_{1}\right) \hat{G}_{ \pm}\left(z_{2}\right) & =\mp 2 z_{12}^{-2} K_{ \pm} \mp z_{12}^{-1} \partial K_{ \pm}\left(z_{2}\right) \\
K_{ \pm}\left(z_{1}\right) G_{\mp}\left(z_{2}\right) & = \pm z_{12}^{-1} \hat{G}_{ \pm}\left(z_{2}\right), \quad K_{ \pm}\left(z_{1}\right) \hat{G}_{\mp}\left(z_{2}\right)=\mp z_{12}^{-1} G_{ \pm}\left(z_{2}\right) \\
K_{3}\left(z_{1}\right) G_{ \pm}\left(z_{2}\right) & = \pm z_{12}^{-1} G_{ \pm}\left(z_{2}\right), \quad K_{3}\left(z_{1}\right) \hat{G}_{ \pm}\left(z_{2}\right)= \pm z_{12}^{-1} \hat{G}_{ \pm}\left(z_{2}\right) \\
K_{l}\left(z_{1}\right) K_{m}\left(z_{2}\right) & =\frac{c}{6} \delta_{l m} z_{12}^{-2}+\epsilon_{l m}{ }^{n} z_{12}^{-1} K_{n}\left(z_{2}\right)
\end{aligned}
$$

where $c=-6(1+\kappa)$.

The interpretation of the above formulas in terms of string theory evident: the bosons $\varphi, \bar{\varphi}$ together with the fermions $\tau, \bar{\tau}$ represent a minimal $N=2$ matter free-field realization, with background charge given by $i \sqrt{\kappa}$. Furthermore, the string ghost-antighost pair corresponds to $\bar{\psi}, \psi$, whereas $\bar{\mu}, \mu$ represents the bosonic ghost-antighost pair of the topological string. After twisting the energy-momentum tensor $T \rightarrow T+\frac{1}{2} \partial K_{3}, \psi$ and $\mu$ acquire spin 2 and $\bar{\psi}$ and $\bar{\mu}$ spin -1 .

The specific realization of the $N=2$ sub-system that we interpret here as matter system is not essential for the closure of the algebra. In fact, it is easy to see that we can replace it by any other realization of the $N=2$ algebra. In contrast to the stringy hamiltonian reductions considered so far, we do not get a Liouville system. This seems to be natural, since as mentioned in the introduction, one would not expect the Liouville field to play an essential role in topological gravity. Let us also note that the only physical observable in equivariant cohomology, namely the generator of gravitational descendents, $\partial \bar{\mu}$, appears here naturally as part of the $s l(2)$ Kac-Moody current $K_{+}$. The central extension of this algebra vanishes and $\partial \bar{\mu}$ decouples from the algebra precisely if $\kappa=-1$ which constitutes the "critical case" $c_{m}=9$ of topological strings.

The screening operators associated with this free-field realization are easily obtained from $\operatorname{sl}(2 \mid 2)$. The following screeners are related to the three simple fermionic roots:

$$
\begin{aligned}
S_{1} & =\frac{1}{2 \pi i} \oint d z \bar{\tau} \exp \left(-\frac{i}{\sqrt{\kappa}} \varphi\right) \\
S_{2} & =\frac{1}{2 \pi i} \oint d z \tau \exp \left(-\frac{i}{\sqrt{\kappa}} \bar{\varphi}\right) \\
S_{3} & =\frac{1}{2 \pi i} \oint d z\left(\psi+\frac{i}{\sqrt{\kappa}} \mu \bar{\tau}\right) \exp \left(-\frac{i}{\sqrt{\kappa}} \varphi\right) .
\end{aligned}
$$


They are needed to define the physical Hilbert space of the theory.

We now turn to discussing the reduction for gradation $I I$. Here, the first-class constraints look

$$
\begin{aligned}
\Phi_{E}^{-}=E^{-}-\frac{\kappa}{2}+\frac{1}{4} \bar{\psi} \bar{\chi} \mu, & \Phi_{J}^{-}=J^{-} \mu, \\
\Phi_{+}^{+-}=j_{+}^{+-}-\psi, & \Phi_{-}^{+-}=j_{-}^{+-}-\chi, \\
\Phi_{+}^{--}=j_{+}^{--}+\frac{1}{2} \mu \bar{\chi}, & \Phi_{-}^{--}=j_{-}^{--}-\frac{1}{2} \mu \bar{\psi} .
\end{aligned}
$$

The action of the constrained theory is now given by

$$
S_{i n v}=\kappa S_{W Z W}[g]+\frac{1}{\pi} \int d^{2} z \operatorname{str}(\bar{A} \Phi)-\frac{1}{2 \pi} \int d^{2} z(\bar{\psi} \bar{\partial} \psi+\bar{\chi} \bar{\partial} \chi-\bar{\mu} \bar{\partial} \mu),
$$

and the OPE's for the auxiliary fields are $\psi\left(z_{1}\right) \cdot \bar{\psi}\left(z_{2}\right)=\chi\left(z_{1}\right) \cdot \bar{\chi}\left(z_{2}\right)=\mu\left(z_{1}\right) \cdot \bar{\mu}\left(z_{2}\right)=z_{12}^{-1}$. The action is invariant under the following gauge transformations:

$$
\begin{array}{rlrl}
\delta \bar{A}=\bar{\partial} \eta+[\bar{A}, \eta], & \delta J & =\partial \eta+[J, \eta] \\
\delta \mu=0, & \delta \bar{\mu}=2 \eta_{J}^{+}+\bar{\chi} \eta_{-}^{++}+\bar{\psi} \eta_{+}^{++}+\frac{1}{2} \eta_{E}^{+} \bar{\psi} \bar{\chi} \\
\delta \psi=\eta_{+}^{++} \mu+\frac{1}{2} \eta_{E}^{+} \bar{\chi} \mu, & \delta \bar{\psi}=-2 \eta_{-}^{-+} \\
\delta \chi=\eta_{-}^{++} \mu-\frac{1}{2} \eta_{E}^{+} \bar{\psi} \mu, & \delta \bar{\chi}=2 \eta_{+}^{-+} .
\end{array}
$$

To quantize the theory, we can use almost the same ghost system as in (8), except that we have to replace the $\left(\beta_{-}^{-+}, \gamma_{+}^{+-}\right)$-system by $\left(\beta_{-}^{+-}, \gamma_{+}^{-+}\right)$, which obeys: $\beta_{-}^{+-}\left(z_{1}\right), \gamma_{+}^{-+}\left(z_{2}\right)=$ $-z_{12}^{-1}$. The ghost currents $j_{+}^{+-}, j_{+}^{-+}, j_{-}^{+-}, j_{-}^{-+}$stay the same as before and therefore can directly be taken over from (12). The other ghost currents get changed, however (notice, e.g., that this time there are no ghost contributions to $J^{ \pm}$):

$$
\begin{aligned}
E_{g h}^{+} & =\frac{1}{2} \beta_{+}^{+-} \gamma_{-}^{++}-\frac{1}{2} \beta_{-}^{+-} \gamma_{+}^{++}, \quad E_{g h}^{-}=-\frac{1}{2} \beta_{+}^{--} \gamma_{-}^{-+}+\frac{1}{2} \beta_{-}^{--} \gamma_{+}^{-+}, \\
E_{g h}^{0} & =-\frac{1}{2} b_{E}^{-} c_{E}^{+}-\frac{1}{4} \beta_{+}^{+-} \gamma_{-}^{-+}-\frac{1}{4} \beta_{+}^{--} \gamma_{-}^{++}+\frac{1}{4} \beta_{-}^{+-} \gamma_{+}^{-+}+\frac{1}{4} \beta_{-}^{--} \gamma_{+}^{++}, \\
J_{g h}^{0} & =\frac{1}{2} b_{J}^{-} c_{J}^{+}+\frac{1}{4} \beta_{+}^{+-} \gamma_{-}^{-+}-\frac{1}{4} \beta_{+}^{--} \gamma_{-}^{++}-\frac{1}{4} \beta_{-}^{+-} \gamma_{+}^{-+}+\frac{1}{4} \beta_{-}^{--} \gamma_{+}^{++}, \\
j_{+g h}^{++} & =-\frac{1}{2} \beta_{-}^{+-} c_{J}^{+} \quad j_{+g h}^{--}=-\frac{1}{2} b{ }_{J}^{-} \gamma_{+}^{-+}, \\
j_{-g h}^{++} & =-\frac{1}{2} \beta_{-}^{+-} c_{J}^{+}, \quad j_{-g h}^{--}=-\frac{1}{2} b_{J}^{-} \gamma_{-}^{-+} .
\end{aligned}
$$

The most drastic difference in comparison to gradation $I$ shows up in the structure of the BRST operator. Using the new gradation to define the complex (13), the BRST charge 
splits into the following five parts:

$$
\begin{aligned}
\mathcal{Q}_{0}= & \frac{1}{2 \pi i} \oint\left(-c_{J}^{+} \mu\right), \quad \mathcal{Q}_{1}=\frac{1}{2 \pi i} \oint\left(-\frac{1}{2} \gamma_{-}^{++} \bar{\chi} \mu-\frac{1}{2} \gamma_{+}^{++} \bar{\psi} \mu\right), \\
\mathcal{Q}_{2}= & \frac{1}{2 \pi i} \oint\left(\frac{\kappa}{2} c_{E}^{+}-\frac{1}{4} c_{E}^{+} \bar{\psi} \bar{\chi} \mu\right), \quad \mathcal{Q}_{3}=\frac{1}{2 \pi i} \oint\left(-\gamma_{-}^{-+} \psi+\gamma_{+}^{-+} \chi\right), \\
\mathcal{Q}_{4}= & \frac{1}{2 \pi i} \oint\left(-c_{E}^{+}\left(E^{-}+\frac{1}{2} E_{g h}^{-}\right)+c_{J}^{+} J^{-}+\gamma_{-}^{-+}\left(j_{+}^{+-}+\frac{1}{2} j_{+g h}^{+-}\right)-\right. \\
& \left.-\gamma_{-}^{++}\left(j_{+}^{--}+\frac{1}{2} j_{+g h}^{--}\right)-\gamma_{+}^{-+}\left(j_{-}^{+-}+\frac{1}{2} j_{-g h}^{+-}\right)-\gamma_{+}^{++}\left(j_{-}^{--}+j_{-g h}^{--}\right)\right),
\end{aligned}
$$

They individually square to zero, and their bigrades are given by $(2,-1),\left(\frac{3}{2}, \frac{1}{2}\right),(1,0)$, $\left(\frac{1}{2}, \frac{1}{2}\right)$ and $(0,1)$. Moreover, they obey the relations $\left\{\mathcal{Q}_{1}, \mathcal{Q}_{3}\right\}+\left\{\mathcal{Q}_{0}, \mathcal{Q}_{4}\right\}=\left\{\mathcal{Q}_{2}, \mathcal{Q}_{3}\right\}+$ $\left\{\mathcal{Q}_{1}, \mathcal{Q}_{4}\right\}=0$. The anti-ghosts $B$ and the constraints $\hat{\Phi}$ form BRST-doublets and we can define a reduced complex, completely parallel to the treatment of gradation $I$. To compute the cohomology on this reduced complex, we note that we can write the BRSToperator as $\mathcal{Q}_{0}+\mathcal{Q}^{\prime}$ with $\mathcal{Q}^{\prime}=\sum_{i=1}^{4} \mathcal{Q}_{i}$. Then this defines a double complex in the weak sense of [16]. Of course, in the spectral sequence we thus have $d_{1}=Q^{\prime}$. But now we can use the splitting given above, such that $Q^{\prime}=Q_{1}+Q^{\prime \prime}$ and $Q^{\prime \prime}=\sum_{i=2}^{4} \mathcal{Q}_{i}$. Proceeding in this manner, it is straightforward to compute the cohomology in a stepwise fashion. Since we use here a rather non-standard decomposition of the BRST-operator, it is useful to denote the non-trivial action of the relevant parts of it on the fields explicitly:

$\mathcal{Q}_{0}: \quad\left[\mathcal{Q}_{0}, J^{0}\right]=\frac{1}{2} c_{J}^{+} \mu, \quad\left[\mathcal{Q}_{0}, \bar{\mu}\right]=-c_{J}^{+}$,

$\mathcal{Q}_{1}: \quad\left[\mathcal{Q}_{1}, \bar{\mu}\right]=-\frac{1}{2} \gamma_{-}^{++} \mu \bar{\chi}-\frac{1}{2} \gamma_{+}^{++} \bar{\psi}, \quad\left\{\mathcal{Q}_{1}, \psi\right\}=-\frac{1}{2} \gamma_{+}^{++} \mu, \quad\left\{\mathcal{Q}_{1}, \chi\right\}=-\frac{1}{2} \gamma_{-}^{++} \mu$, $\left[\mathcal{Q}_{1}, \hat{E}^{0}\right]=\frac{1}{8} \gamma_{-}^{++} \bar{\chi} \mu+\frac{1}{8} \gamma_{+}^{++} \bar{\psi} \mu, \quad\left[\mathcal{Q}_{1}, \hat{J}^{0}\right]=\frac{1}{8} \gamma_{-}^{++} \bar{\chi} \mu+\frac{1}{8} \gamma_{+}^{++} \bar{\psi} \mu$, $\left\{\mathcal{Q}_{1}, \hat{j}_{+}^{-+}\right\}=-\frac{1}{4} c_{J}^{+} \bar{\chi} \mu, \quad\left\{\mathcal{Q}_{1}, \hat{j}_{-}^{-+}\right\}=\frac{1}{4} c_{J}^{+} \bar{\chi} \mu$,

$\mathcal{Q}_{2}: \quad\left[\mathcal{Q}_{2}, \bar{\mu}\right]=-\frac{1}{4} c_{E}^{+} \bar{\psi} \bar{\chi}, \quad\left\{\mathcal{Q}_{2}, \psi\right\}=\frac{1}{4} c_{E}^{+} \bar{\chi} \mu, \quad\left\{\mathcal{Q}_{2}, \chi\right\}=-\frac{1}{4} c_{E}^{+} \bar{\psi} \mu$, $\left[\mathcal{Q}_{2}, \hat{E}^{0}\right]=-\frac{\kappa}{4} c_{E}^{+}-\frac{1}{8} c_{E}^{+} \bar{\chi} \bar{\psi} \mu, \quad\left\{\mathcal{Q}_{2}, \hat{j}_{+}^{-+}\right\}=\frac{\kappa}{4} \gamma_{+}^{++}+\frac{1}{8} \gamma_{+}^{++} \bar{\chi} \bar{\psi} \mu$, $\left\{\mathcal{Q}_{2}, \hat{j}_{-}^{-+}\right\}=\frac{\kappa}{4} \gamma_{-}^{++}+\frac{1}{8} \gamma_{-}^{++} \bar{\chi} \bar{\psi} \mu$,

$\mathcal{Q}_{3}: \quad\left\{\mathcal{Q}_{3}, \bar{\psi}\right\}=-\gamma_{-}^{-+}, \quad\left\{\mathcal{Q}_{3}, \bar{\chi}\right\}=\gamma_{+}^{-+}, \quad\left\{\mathcal{Q}_{3}, \hat{j}_{+}^{++}\right\}=\frac{1}{2} c_{J}^{+} \psi$, $\left\{\mathcal{Q}_{3}, \hat{j}_{-}^{++}\right\}=\frac{1}{2} c_{J}^{+} \chi, \quad\left[\mathcal{Q}_{3}, \hat{E}^{+}\right]=\frac{1}{2} \gamma_{-}^{++} \psi-\frac{1}{2} \gamma_{+}^{++} \chi$, $\left[\mathcal{Q}_{3}, \hat{E}^{0}\right]=-\frac{1}{4} \gamma_{-}^{-+} \psi+\frac{1}{4} \gamma_{+}^{-+} \chi, \quad\left[\mathcal{Q}_{3}, \hat{J}^{0}\right]=\frac{1}{4} \gamma_{-}^{-+} \psi-\frac{1}{4} \gamma_{+}^{-+} \chi$.

From these equations we immediately see that $\left(\bar{\mu}, c_{J}^{+}\right)$decouple from the $\mathcal{Q}_{0}$-cohomology. Then, looking at $\mathcal{Q}_{1}$, we note that the auxiliary fields $(\psi, \chi)$ lie in BRST-doublets together 
with the composite operators $\left(\gamma_{+}^{++} \mu, \gamma_{-}^{++} \mu\right)$. This means that these decouple from the $\mathcal{Q}_{1}$-cohomology. In the next step we have to take into account that in the complex $E_{2}=H^{*}\left(\hat{\mathcal{A}} ; \mathcal{Q}_{1}\right)$ there are vanishing relations of the form $\gamma_{+}^{++} \mu=\gamma_{-}^{++} \mu \sim 0$. Therefore we find that the composite fields $\hat{j}_{+}^{-+} \mu$ and $\hat{j}_{-}^{-+} \mu$ are non-trivial elements of the $\mathcal{Q}_{2^{-}}$ cohomology on $E_{2}$. Proceeding further one finds that the spectral sequence collapses after the fourth step and that the cohomology of $\mathcal{Q}$ is spanned by $\left\{\hat{E}^{+}, \hat{J}^{+}, \hat{J}^{0}+\frac{1}{2} \mu \bar{\mu}-\right.$ $\left.\frac{1}{4} \psi \bar{\psi}-\frac{1}{4} \chi \bar{\chi}, \hat{j}_{+}^{++}, \hat{j}_{-}^{++}, \hat{j}_{+}^{-+} \mu, \hat{j}_{-}^{-+} \mu, \mu\right\}$. It is surprising that not all the elements that lie in $\operatorname{ker}\left(a d_{E^{+}}\right)$correspond to leading terms in the cohomology. It is however not difficult to see, by following a generalized tic-tac-toe procedure, that one picks up the highest weight auxiliaries $\psi, \chi$ in the tail of $\hat{j}_{+}^{-+} \mu$ and $\hat{j}_{-}^{-+} \mu$. It is also clear that the truncation to zero grade fields still provides an algebra isomorphism.

In order to end up with a string-type free-field realization, we demand that one of the supercharges is given by just a single auxiliary field. We could choose $\chi$, or, equally well, $\psi$. To be specific, let us choose $\chi$, and perform the following similarity transformation:

$$
S=\exp \left[-\frac{1}{2 \pi i} \oint d z\left(\frac{1}{\kappa} \bar{\chi} \hat{E}^{0} \bar{\psi} \mu+\frac{1}{2} \bar{\chi} \partial \bar{\psi} \mu+\frac{1}{\kappa} \bar{\chi} \hat{J}^{0} \bar{\psi} \mu\right)\right] .
$$

Furthermore, we bosonize the Cartan currents:

$$
\hat{E}^{0}=\frac{i \sqrt{\kappa}}{2 \sqrt{2}} \partial \varphi_{1}, \quad \hat{J}^{0}=\frac{\sqrt{\kappa}}{2 \sqrt{2}} \partial \varphi_{2}, \quad \varphi_{i}\left(z_{1}\right) \cdot \varphi_{j}\left(z_{2}\right)=-\delta_{i j} \ln \left(z_{12}\right)
$$

Then, finally, we arrive at a second free-field realization of the small $N=4$ algebra that can attributed to topological string theory:

$$
\begin{aligned}
T= & -\frac{1}{2}\left(\partial \varphi_{1}\right)^{2}+\frac{i(1-\kappa)}{\sqrt{2 \kappa}} \partial^{2} \varphi_{1}-\frac{1}{2}\left(\partial \varphi_{2}\right)^{2}+\frac{1}{\sqrt{2 \kappa}} \partial^{2} \varphi_{2}+ \\
& +\mu \partial \bar{\mu}-\frac{3}{2} \chi \partial \bar{\chi}-\frac{1}{2} \partial \chi \bar{\chi}-\frac{3}{2} \psi \partial \bar{\psi}-\frac{1}{2} \partial \psi \bar{\psi} \\
G_{+}= & -\bar{\psi}\left(-\frac{1}{2}\left(\partial \varphi_{1}\right)^{2}+\frac{i(1-\kappa)}{\sqrt{2 \kappa}} \partial^{2} \varphi_{1}-\frac{1}{2}\left(\partial \varphi_{2}\right)^{2}+\frac{1+\kappa}{\sqrt{2 \kappa}} \partial^{2} \varphi_{2}-\psi \partial \bar{\psi}\right)+ \\
& +\sqrt{2 \kappa} \partial\left(\bar{\psi} \partial \varphi_{1}\right)-\frac{1-2 \kappa}{2} \partial^{2} \bar{\psi}+\chi \bar{\mu} \\
G_{-}= & -\psi-\bar{\chi} \partial \mu-2 \partial \bar{\chi} \mu \\
\hat{G}_{+}= & \bar{\chi}\left[-\frac{1}{2}\left(\partial \varphi_{1}\right)^{2}+\frac{i(1-\kappa)}{\sqrt{2 \kappa}} \partial^{2} \varphi_{1}-\frac{1}{2}\left(\partial \varphi_{2}\right)^{2}+\frac{1+\kappa}{\sqrt{2 \kappa}} \partial^{2} \varphi_{2}+\right. \\
& \left.-2 \psi \partial \bar{\psi}-\partial \psi \bar{\psi}+\mu \partial \bar{\mu}+\frac{1}{2} \partial \mu \bar{\mu}-\chi \partial \bar{\chi}\right]- \\
& -\bar{\mu}\left(\psi+\frac{1}{2} \bar{\chi} \partial \mu+\partial \bar{\chi} \mu\right)-\partial\left[\bar{\chi}\left(\sqrt{2 \kappa} \partial \varphi_{2}\right)-\psi \bar{\psi}+\mu \bar{\mu}\right]-(1+\kappa) \partial^{2} \bar{\chi} \\
\hat{G}_{-}= & \chi \\
K_{+}= & \frac{1}{2} \bar{\chi} \bar{\psi}\left(\partial \varphi_{1}\right)^{2}+\frac{1}{2} \bar{\chi} \bar{\psi}\left(\partial \varphi_{2}\right)^{2}-\frac{i(1-\kappa)}{\sqrt{2 \kappa}} \bar{\chi} \bar{\psi} \partial^{2} \varphi_{1}-\frac{1-\kappa}{\sqrt{2 \kappa}} \bar{\chi} \bar{\psi} \partial^{2} \varphi_{2}-\bar{\chi} \partial \bar{\psi} \partial \varphi_{2}+
\end{aligned}
$$




$$
\begin{aligned}
& +\bar{\mu} \chi \bar{\chi}-\bar{\mu}^{2} \mu-\sqrt{2 \kappa} \bar{\mu} \partial \varphi_{2}+\bar{\mu} \psi \bar{\psi}+\bar{\chi} \bar{\psi} \psi \partial \bar{\psi}-\frac{1-\kappa}{2} \bar{\chi} \partial^{2} \bar{\psi}-(1+\kappa) \partial \bar{\mu} \\
K_{3}= & \sqrt{2 \kappa} \partial \varphi_{2}-\chi \bar{\chi}-\psi \bar{\psi}+2 \mu \bar{\mu} \\
K_{-}= & \mu
\end{aligned}
$$

After twisting, the auxiliary fields, $(\psi, \chi, \mu)$ have spin 2 and $(\bar{\psi}, \bar{\chi}, \bar{\mu})$ have spin -1 . From the form of the generators we see that we can indeed interpret $(\chi, \bar{\chi})$ and $(\mu, \bar{\mu})$ as the ghosts of the topological string. The rest constitutes the matter sector of the topological string and is isomorphic to a particular realization of the ordinary bosonic string! That is, $\varphi_{1}$ is the matter and $\varphi_{2}$ the Liouville system of the bosonic string, and the diffeomorphism ghosts are given by $(\psi, \bar{\psi})$. Indeed, modulo the terms arising from the topological ghostsystem, the currents $T, G_{+}, G_{-}$and $K_{3}$ are exactly the well-known $N=2$-currents of the bosonic string [1, 2]. This means that the above formulas should be interpreted as an embedding of the non-critical bosonic string into the topological string. Note that the critical topological string, which corresponds to $\kappa=-1$, coincides with the critical bosonic string, as can be seen by the vanishing of the background charges.

For sake of completeness, we present the following screening operators,

$$
\begin{aligned}
& S_{1}=\frac{1}{2 \pi i} \oint d z \exp \left(-i \sqrt{2 \kappa} \varphi_{1}\right) \\
& S_{2}=\frac{1}{2 \pi i} \oint d z \psi \exp \left[-\frac{i}{\sqrt{2 \kappa}}\left(\varphi_{1}-i \varphi_{2}\right)\right] \\
& S_{3}=\frac{1}{2 \pi i} \oint d z(\chi+2 \mu \partial \bar{\psi}+\partial \mu \bar{\psi}) \exp \left[\frac{-i}{\sqrt{2 \kappa}}\left(\varphi_{1}-i \varphi_{2}\right)\right]
\end{aligned}
$$

that are necessary to properly define the free-field Hilbert space.

Generalizing the above considerations to topological $W_{n}$-strings [12], one expects that a reduction of $\operatorname{sl}(n \mid n)$ should be relevant. Let us consider the reduction of $\operatorname{sl}(3 \mid 3)$ in some more detail. As we want to have an $N=2 W_{3}$ algebra as a part of the full string BRST algebra, we need to analyze the embedding of $s l(3 \mid 2)$ into $s l(3 \mid 3)$. The embedding of $s l(2 \mid 1)$ in $s l(3 \mid 2)$ is then precisely the one that gives rise to the $N=2 W_{3}$ algebra. In terms of $s l(2)$ representations, we find that the bosonic part of $\operatorname{sl}(3 \mid 3)$ will give rise to a spin 3 , two spin 2 , two spin $\frac{3}{2}$ and one spin 1 current. The fermionic part yields two sets of currents with spins $\frac{5}{2}, 2$ and $\frac{3}{2}$. Thus we find 6 bosonic and 6 fermionic generators. However, from a $W_{3}$ extension of the small $N=4$ algebra one expects 8 bosonic and 8 fermionic generators. We thus see from this simple counting argument that the reduction of $\operatorname{sl}(3 \mid 3)$ will not give rise to a naive formulation of topological $W_{3}$-gravity [12].

It is nevertheless worth trying to go on with the analysis. It is easy to find a gradation analogous to the one we used for $\operatorname{sl}(2 \mid 2)$, where two of the $\operatorname{sl}(2)$ highest weights, one belonging to a fermionic triplet and one belonging to a bosonic doublet, have negative 
grades. From our discussion of $\operatorname{sl}(2 \mid 2)$ it is clear that this will result in bosonic and fermionic ghost systems. Under the $U(1)$-current of $s l(3 \mid 3)$ (the one which corresponds to the ghost current after the reduction), these two ghosts systems have charges 1 and $\frac{3}{2}$, respectively, such that both would obtain spin 3 in the twisted superconformal algebra. They should therefore represent the high-spin ghost-systems of topological $W_{3}$-gravity. We thus expect that one obtains from such a reduction topological $W_{3}$-gravity to be realized in a kind of "half-rotated" matter picture, where the low-spin ghosts are decoupled from the theory. That such a realization exists is in fact quite plausible since we know that the spectrum of topological $W_{3}$-gravity can be represented entirely in the matter sector [12].

Combining the results of this paper with [3], we are drawn to conclude that a very systematic and almost completely algebraic approach to string theory might be attainable, at least as far as controlling and classifying the possible gauge structures on the worldsheet goes. Indeed, these two papers demonstrate that all known string theories can be obtained in a straightforward way from reducing WZW models. As all simple supergroups have been classified, the natural step to take now is a complete classification of string theories that can be obtained this way. This is presently under investigation [20]. We hope that such a group theoretical approach to string theory will eventually provide clues to the structure of a "universal" string field theory that would have vacua corresponding to arbitrary gauge groups on the world-sheet.

Acknowledgements We extensively used Kris Thielemans Mathematica package OPEdefs.m [21] for the computations.

\section{References}

[1] B. Gato-Rivera and A. M. Semikhatov Phys. Lett. B293 (1992) 72, hep-th/9207004

[2] M. Bershadsky, W. Lerche, D. Nemeschansky and N. P. Warner, Nucl. Phys. B401 (1993) 304, hep-th/9211040

[3] A. Boresch, K. Landsteiner, W. Lerche and A. Sevrin, Superstrings from Hamiltonian Reduction, preprint CERN-TH 7370/94, hep-th/9408033, to appear in Nucl. Phys. $\mathrm{B}$

[4] K. Ito and H. Kanno, Mod. Phys. Lett. A9 (1994) 1377; Lie Superalgebra and Extended Topological Conformal Symmetry in Non-critical $W_{3}$ Strings, preprint UTHEP-277, hep-th/9405049

[5] M. Bershadsky and H. Ooguri, Commun. Math. 126 (1989) 49 
[6] A. M. Semikhatov, Talk given at the $28^{\text {th }}$ International Symposium on the Theory of Elementary Particles, Wendisch-Rietz, August 30 - September 3, 1994, hep-th/9410109

[7] J. Distler, Nucl. Phys. B342 (1990) 523

[8] E. Verlinde and H. Verlinde, Nucl. Phys. B352 (1991) 1234

R. Dijkgraaf, E. Verlinde and H. Verlinde, Nucl. Phys. B352 (1991) 59

[9] E. Witten Chern Simons Gauge Theory as a String Theory, preprint IASSNS-HEP92-45, hep-th/9207094

M. Bershadsky, S. Cecotti, H. Ooguri and C. Vafa, Commun. Math. Phys. Vol. 165 No. 2 (1994), 311

[10] N. Berkovits and C. Vafa, Mod. Phys. Lett. A9 (1994) 653

[11] F. Bastianelli and N. Otha, Phys. Rev. Lett. 73 (1994) 1199

[12] W. Lerche and A. Sevrin, Nucl. Phys. B428 (1994) 259, hep-th/9403183

[13] A. Sevrin, W. Troost and A. Van Proeyen, Phys. Lett. B208 (1988) 447

[14] M. Ademollo et al., Phys. Lett. B62 (1976) 105; Nucl. Phys. B111 (1976) 77

[15] A. Sevrin, K. Thielemans and W. Troost, Nucl. Phys. B407 (1993) 459, hep-th/9303133

[16] A. Sevrin and W. Troost, Phys. Lett. B315 (1993) 304, hep-th/9306033; J. de Boer and T. Tjin, Comm. Math.Phys. 160 (1994) 317, hep-th/9302006

[17] V. G. Kac, Comm. Math. Phys. 53 (1977) 31

L. Frappat, A. Sciarrino and P. Sorba, Comm. Math. Phys. 121 (1989) 457

Cornwall, Group Theory in Physics Vol.3

[18] T. Eguchi, H. Kanno, Y. Yamada and S.-K. Yang, Pys. Lett. B305 (1993) 235, hep-th/9302048

[19] T. Eguchi and S.K. Yang, Mod. Phys. Lett. A5 (1990), 1693

[20] E. Ragoucy, A. Sevrin and P. Sorba, in preparation

[21] K. Thielemans, Int. J. Mod. Phys. C Vol. 2, No. 3, (1991) 787 